\title{
Abstract: Studying Robustness of Semantic Segmentation under Domain Shift in Cardiac MRI
}

\author{
Peter M. Full ${ }^{1,2}$, Fabian Isensee ${ }^{1}$, Paul F. Jäger ${ }^{1}$, Klaus Maier-Hein ${ }^{1}$ \\ ${ }^{1}$ Division of Medical Image Computing, German Cancer Research Center (DKFZ), \\ Heidelberg, Germany \\ ${ }^{2}$ Medical Faculty Heidelberg, Heidelberg University, Heidelberg, Germany \\ p.full@dkfz-heidelberg.de
}

Cardiac magnetic resonance imaging (cMRI) is an integral part of diagnosis in many heart related diseases. Recently, deep neural networks (DNN) have demonstrated successful automatic segmentation, thus alleviating the burden of time-consuming manual contouring of cardiac structures. Moreover, frameworks such as nnU-Net provide entirely automatic model configuration to unseen datasets enabling out-of-the-box application even by non-experts. However, current studies commonly neglect the clinically realistic scenario, in which a trained network is applied to data from a different domain such as deviating scanners or imaging protocols. This potentially leads to unexpected performance drops of deep learning models in real life applications. In this work, we systematically study challenges and opportunities of domain transfer across images from multiple clinical centres and scanner vendors. In order to maintain out-of-the-box usability, we build upon a fixed U-Net architecture configured by the nnU-net framework to investigate various data augmentation techniques and batch normalization layers as an easy-to-customize pipeline component and provide general guidelines on how to improve domain generalizability abilities in existing deep learning methods. Our proposed method ranked first at the Multi-Centre, Multi-Vendor \& Multi-Disease Cardiac Image Segmentation Challenge (MEMs). We expect our experimental insights to be helpful for clinical scenarios in which externally trained DNNs are deployed on in-house data with potentially differing scanner vendors or imaging protocols. In such scenarios, our approach is able to improve segmentation quality by bridging domain gaps between data sets and as a consequence reduces time-efforts and costs associated with generating manually annotated in-domain datasets at clinical sites. [1]

\section{References}

1. Full PM, Isensee F, Jäger PF, et al. Studying robustness of semantic segmentation under domain shift in cardiac MRI. STACOM. 2020;. 